\title{
Efeito do sevofluorano sobre a freqüência cardíaca fetal no terço final de gestação de cadelas
}

\author{
[Effect of sevoflurane on fetal heart rate in last third pregnant bitches] \\ L.M. Matsubara ${ }^{1}$, V.N.L.S. Oliva ${ }^{2}$, D.T. Gabas ${ }^{1}$, L. Bevilacqua ${ }^{1}$ \\ L. Rodello ${ }^{1}$, S.H.V.Perri ${ }^{2}$ \\ ${ }^{1}$ Aluno de pós-graduação - FMZ-UNESP - Botucatu, SP \\ ${ }^{2}$ Curso de Medicina Veterinária - UNESP - Araçatuba, SP \\ Rua Clóvis Pestana, 793 \\ 16050-680 - Araçatuba, SP
}

\begin{abstract}
RESUMO
Estudaram-se os efeitos do sevofluorano sobre a freqüência cardíaca nos fetos de cadelas no terço final de gestação, mediante a mensuração da freqüência cardíaca fetal com a utilização do ultra-som. Nove cadelas, sem raça definida, entre um e cinco anos de idade, com aproximadamente 45 dias de gestação, foram anestesiadas com acepromazina $(0,05 \mathrm{mg} / \mathrm{kg}$, IV), propofol $(5 \mathrm{mg} / \mathrm{kg}$, IV) e sevofluorano. O monitoramento da freqüência cardíaca fetal foi realizado antes da medicação pré-anestésica (M0), 15 minutos após a intubação traqueal (M1), aos 30 minutos (M2) e 60 minutos (M3) do período de manutenção anestésica. A pressão arterial sistólica (PAS), média (PAM) e diastólica (PAD) foram obtidas pelo método não invasivo, sendo a PAM avaliada também pelo método invasivo. Por meio do monitoramento da freqüência cardíaca fetal média não se observou diferença significativa entre M0, M1 e M2, e verificou-se elevação apenas aos 60 minutos da manutenção anestésica (M3) em relação ao M0, porém sem significado clínico. O protocolo anestésico provocou diminuição significativa da pressão sangüínea arterial materna sem alterar, porém a freqüência cardíaca dos fetos.
\end{abstract}

Palavras-chave: cadela, feto, anestesia inalatória, ultra-som, gestação

\begin{abstract}
The effect of sevoflurane on fetal heart rate of 45-day fetuses was investigated through monitoring fetal heart rate by ultrasonographic evaluation in nine adult bitches aging from 1 to 5 -year-old. After sedation with acepromazine $(0.05 \mathrm{mg} / \mathrm{kg}, \mathrm{IV})$, the anesthetic induction was accomplished using propofol $(5 \mathrm{mg} / \mathrm{kg}$, $I V)$ and the maintenance was kept with sevoflurane. The fetal heart rate was measured before sedation (M0), 15 minutes after endotracheal intubation (M1), at 30 (M2) and 60 minutes (M3) from the beginning of general anesthesia. The systolic, diastolic and mean blood pressures were obtained by indirect techniques, while mean blood pressure were also obtained by direct technique. The measure of fetal heart rate did not show any difference between $M 0, M 1$ and $M 2$, but there was a remarkable increase in the last moment of the study (M3) comparing to M0, however without clinical relevance. The anesthetic protocol employing acepromazine, propofol and sevoflurane in 45-day pregnancy bitches decreased their arterial blood pressure without affecting the fetal heart rate.
\end{abstract}

Keywords: bitch, fetuses, inhalation anesthesia, ultrasonographic, pregnancy

Recebido em 4 de maio de 2005

Aceito em 23 de novembro de 2006

E-mail: matsubara@fmvz.unesp.br

Apoio: FAPESP 


\section{INTRODUÇÃO}

A anestesia em pacientes gestantes é um desafio para os anestesistas que devem procurar utilizar fármacos que promovam depressão mínima da mãe e dos fetos. É importante ressaltar que durante a gestação e no momento do parto ocorrem alterações fisiológicas que interferem na qualidade da anestesia (Pascoe e Moon, 2001).

A anestesia epidural está associada a índices baixos de mortalidade fetal, porém essa técnica necessita de treinamento. Além disso, os anestésicos locais produzem paralisia de membros pélvicos e, portanto, aumentam o tempo necessário para a fêmea se recuperar e cuidar dos seus filhotes (Funkquist et al., 1997). Esses autores verificaram que a taxa de viabilidade fetal foi similar no protocolo com propofol e isofluorano $(70 \%)$ e epidural $(72 \%)$ sendo, porém, mais alta que no protocolo com a utilização do tiopental (34\%).

Benson e Thurmon (1984) compararam a anestesia inalatória com a epidural e afirmaram que as vantagens da inalatória são a velocidade $\mathrm{e}$ a facilidade de indução e o controle do plano anestésico. Mais ainda, a intubação traqueal assegura o controle das vias respiratórias e permite a administração de oxigênio, prevenindo, ainda, a aspiração de vômito, porém causa maior depressão neonatal.

Todos os fármacos utilizados na anestesia ultrapassam a barreira placentária; a única exceção são os bloqueadores neuromusculares, sendo, por isso impossível anestesiar a mãe de maneira seletiva sem deprimir o feto (Benson e Thurmon, 1987). A permeabilidade da placenta está relacionada à sua classificação e às propriedades físico-químicas dos fármacos (Hall et al., 2001).

Os anestésicos inalatórios provocam depressão fetal que é diretamente proporcional ao plano anestésico da mãe. Portanto, é importante manter a anestesia em um plano adequado para o procedimento cirúrgico, evitando-se níveis profundos que causam hipotensão materna e diminuição do fluxo sangüíneo uterino, com hipóxia e acidose fetal (Thurmon et al., 1996).

Os valores obtidos da pressão arterial pelo método invasivo são mais fidedignos em todas as pressões mensuradas, inclusive em pacientes hipotensos (Nunes, 2002). Apesar dessas vantagens, a avaliação da pressão arterial pelo método não invasivo tem sido freqüentemente utilizada, por ser um método mais confortável para o paciente. Entre as desvantagens do método não invasivo encontrou-se a falta de precisão na avaliação da pressão arterial diastólica, especialmente em pacientes hipotensos (Nunes, 2002).

A realização do exame ultra-sonográfico para a aferição da freqüência cardíaca fetal (FCf) é um ótimo recurso para avaliar a viabilidade fetal, pois no feto e no neonato o débito cardíaco depende principalmente da freqüência cardíaca, pelo fato de o sistema nervoso simpático ser pouco desenvolvido nessa idade (Pascoe e Moon, 2001). A FCf acima de 200bpm é um bom indicativo de que o valor está dentro dos padrões esperados para a espécie canina, no entanto, freqüência mais baixa pode ser indicativa de débito cardíaco inadequado, sugerindo a necessidade de intervenção (Pascoe e Moon, 2001).

O objetivo deste estudo foi avaliar os efeitos do protocolo anestésico sobre a freqüência cardíaca de fetos com aproximadamente 45 dias de idade, por meio do ultra-som.

\section{MATERIAL E MÉTODOS}

Nove cadelas, sem raça definida, com peso médio de $15 \mathrm{~kg}$ e idade entre 1 e 5 anos, foram submetidas a um período de acompanhamento reprodutivo, que constituiu da determinação do período de pró-estro, definido mediante visualização do sangramento vaginal e edema vulvar, ao qual seguiu-se o estudo da citologia vaginal para que se obtivesse a determinação do início do período estral. A cobrição das cadelas foi realizada por monta natural ou inseminação artificial, a última adotada caso a cadela não aceitasse o macho. O diagnóstico de gestação foi realizado no $30^{\circ}$ dia após a cobrição por meio de exame ultra-sonográfico.

O momento de submeter a cadela ao procedimento anestésico (45 dias de gestação) foi definido conforme técnica descrita por Feldman e Nelson (1996), que preconiza a realização de radiografia abdominal da mãe, na 
qual é possível determinar o número de fetos existentes, pois o crânio e a coluna tornam-se radiopacos entre 44 e 46 dias, e, também, pela determinação do primeiro dia de diestro e subseqüente contagem dos dias requeridos.

No dia do procedimento anestésico as cadelas foram submetidas a exames pré-anestésicos, com o objetivo de avaliar o estado de higidez. Os animais foram tranqüilizados com acepromazina $0,2 \%{ }^{1}$ na dose de $0.05 \mathrm{mg} / \mathrm{kg}$ IV. A veia cefálica foi cateterizada com os animais em decúbito lateral; decorridos 15 minutos, o propofol ${ }^{2}$ foi administrado na dose de $5 \mathrm{mg} / \mathrm{kg} \mathrm{IV}$, durante um minuto, para evitar a ocorrência de apnéia.

Após a perda total do reflexo palpebral e laringotraqueal, nas cadelas posicionadas em decúbito dorsal, efetuou-se a intubação orotraqueal $^{3}$. Iniciou-se a fase de manutenção da anestesia com a conexão da sonda endotraqueal ao sistema circular de anestesia ${ }^{4}$ e a vaporização do sevofluorano ${ }^{2}$, diluído em oxigênio ${ }^{5}$, por meio do vaporizador calibrado ${ }^{6}$, em fluxo de 1 a $1,51 / \mathrm{min}$, em média. Utilizou-se a concentração de anestésico necessária para manter o segundo plano do III estágio de anestesia, segundo a classificação de Guedel (1951).

Após o início da vaporização do anestésico, inseriu-se um cateter $20 \mathrm{~g}^{7}$ na artéria femoral, que foi conectado a um manômetro analógico ${ }^{8}$, para medir a pressão arterial média por método invasivo e compará-la com a pressão obtida por meio do método não-invasivo.

A pressão arterial (PA), obtida pelo método indireto ${ }^{9}$, foi avaliada com o manguito colocado na base da cauda do animal, obtendo-se, dessa forma, a pressão arterial sistólica (PAS), a pressão arterial média (PAM) e a pressão arterial diastólica (PAD), mensuradas antes da medicação pré-anestésica (MPA) (M0), 15

\footnotetext{
${ }^{1}$ Univet S.A. Indústria Veterinária, São Paulo - SP

${ }^{2}$ Cristália Produtos Químicos e Farmacêuticos Ltda, São

Paulo- SP

${ }^{3}$ Sonda endotraqueal de Maggil - Rüsh - Alemanha

${ }^{4}$ Aparelho Oxigel modelo 800 -Oxigel Materiais

Hospitalares Ind.Com.Ltda., São Paulo-SP

${ }^{5}$ White-Martins do Brasil, São Paulo, SP

${ }^{6}$ Vaporizador calibrado para Sevofluorano 1225- K Takaoka, São Paulo- SP

${ }^{7}$ Insyte - BD, Utah -EUA

${ }^{8}$ Manômetro Heidji, São Paulo-SP

${ }^{9}$ Monitor de pressão arterial -Dixtal - DX 2710
}

minutos após a intubação traqueal (M1), e aos 30 (M2) e 60 minutos (M3) da manutenção anestésica. Foram realizadas três aferições consecutivas desses parâmetros, calculando-se a média aritmética para a determinação do valor final da PAS, PAM e PAD.

A freqüência cardíaca fetal (FCf) foi obtida por meio de exame ultrassonográfico, utilizando-se de um aparelho com transdutor convexo de $5 \mathrm{mgHz}^{10}$, dividindo-se o abdômen em quatro quadrantes e identificando-se quatro fetos diferentes. A contagem foi realizada por, no mínimo, duas pessoas, durante 30 segundos, três vezes em cada feto. Dos valores de FCf obtidos, obteve-se a média aritmética para se determinar o valor da FCf média. Este parâmetro foi avaliado em M0, M1, M2 e M3.

Os dados foram submetidos à análise de variância com medidas repetidas e análise dos resíduos para verificar a normalidade e homogeneidade de variâncias, pré-requisitos necessários para a análise e ao teste Tukey $(\mathrm{P}<0,05)$ (Zar, 1999).

$\mathrm{Na}$ comparação entre a pressão arterial média obtida pelos métodos não-invasivo e invasivo usou-se $\mathrm{o}$ teste $\mathrm{t}$ pareado. Os valores foram expressos em média \pm erro padrão da média (EPM), com nível de significância de 5\%.

As análises estatísticas foram feitas empregandose o Statistic Analysis System.

\section{RESULTADOS E DISCUSSÃO}

A diminuição da PAS, PAD e PAM após a MPA e a indução anestésica (Tab. 1) estão de acordo com os resultados de Gonçalves (2003), que concluiu que todas as fenotiazinas causam discreta hipotensão na espécie canina, independente da dose utilizada.

A acepromazina é um potente bloqueador dos receptores alfa-adrenérgicos e depressor dos centros vasomotores centrais, sendo que esses dois efeitos contribuem para a hipotensão durante a anestesia geral (Farver et al., 1986; Brock, 1994).

\footnotetext{
${ }^{10}$ Ultra-som Aloka -modelo SSD-210 DXII, São Paulo-SP
} 
Tabela 1. Valores médios e erro padrão da média da pressão arterial sistólica (PAS), da pressão arterial média obtida pelo método não invasivo (PAM ninv) e invasivo (PAM inv), da pressão arterial diastólica (PAD) e da freqüência cardíaca fetal (FCfm) em cadelas anestesiadas pelo sevofluorano, pré-tratadas com acepromazina $(0,05 \mathrm{mg} / \mathrm{kg})$ e propofol $(5 \mathrm{mg} / \mathrm{kg})$ aos 45 dias de gestação, de acordo com os momentos

\begin{tabular}{ccccc}
\hline & M0 & M1 & M2 & M3 \\
\hline PAS & $122 \pm 4 \mathrm{a}$ & $84 \pm 3 \mathrm{c}$ & $87 \pm 3 \mathrm{bc}$ & $98 \pm 4 \mathrm{~b}$ \\
PAM ninv & $83 \pm 5 \mathrm{a}$ & $56 \pm 3 \mathrm{bcA}$ & $56 \pm 3 \mathrm{bcB}$ & $66 \pm 3 \mathrm{cA}$ \\
PAM inv & $\mathrm{NM}$ & $62 \pm 3 \mathrm{aA}$ & $63 \pm 3 \mathrm{aA}$ & $71 \pm 4 \mathrm{aA}$ \\
PAD & $54 \pm 5 \mathrm{a}$ & $37 \pm 3 \mathrm{~b}$ & $36 \pm 2 \mathrm{~b}$ & $44 \pm 3 \mathrm{~b}$ \\
FCfm & $182 \pm 3 \mathrm{~b}$ & $186 \pm 5 \mathrm{ab}$ & $187 \pm 6 \mathrm{ab}$ & $191 \pm 4 \mathrm{a}$ \\
\hline
\end{tabular}

Médias seguidas por letras distintas minúsculas na linha e maiúsculas na coluna diferem entre si pelo teste Tukey $(\mathrm{P}>0,05)$. NM: variável não mensurada

M0= medicação pré_anestésica, M1= medicação 15min após intubação traqueal, M2 e M3= medicação aos 30 e 60 min da manutenção anestésica.

Houve redução da PAS em todos os momentos da manutenção anestésica, sendo os valores obtidos em M1, M2 e M3 significativamente mais baixos que o obtido em M0. Resultados semelhantes foram relatados por Probst e Webb (1983), que observaram diminuição da PAS obtida pelo método invasivo, em cadelas próximas ao momento do parto, porém anestesiadas com halotano e induzidas com tiamilal sódico, sem a aplicação da MPA. A redução da PAS é justificada pela diminuição da resistência vascular (Probst e Webb, 1983).

A pressão arterial média mensurada pelo método não-invasivo reduziu-se durante todo o procedimento anestésico, com valores sempre inferiores a 70mmHg, caracterizando-se hipotensão. Este fato decorre de alterações fisiológicas da gestação (Brooks e Keil, 1994) associada à administração de fármacos anestésicos hipotensores (Brock, 1994; Thurmon et al., 1996). Segundo Kazama e Ikeda (1985), essa redução é proporcional à concentração do sevofluorano administrado. Uma das provavéis causas da queda da PAM é a diminuição da resistência vascular periférica (Bernard et al., 1990; Mutoh et al., 1997) decorrente do efeito do sevofluorano.

A mensuração da PAM de maneira invasiva foi realizada somente no período de manutenção anestésica e, neste período, não ocorreram diferenças entre os valores obtidos. Contudo, ao se comparar os valores da PAM obtida pelos métodos não-invasivo e invasivo, observaram-se resultados significativamente mais baixos no método não-invasivo somente em M2 (Tab. 1 ).
Nunes (2002) afirmou que o método não invasivo de medida da pressão arterial não é preciso ao se avaliar a $\mathrm{PAD}$, especialmente em paciente hipotensos, fato não comprovado neste estudo devido à impossibilidade de aferição desse parâmetro pelo método invasivo, o que não permitiu uma comparação entre os dois métodos.

$\mathrm{Na}$ mulher gestante, o decúbito dorsal pode resultar em compressão da veia cava caudal e da artéria aorta devido ao aumento do volume uterino, que diminui o retorno venoso e o débito cardíaco, resultando em hipotensão e conseqüente diminuição do fluxo sangüíneo uterino e renal (Thurmon et al., 1996). Por isso, as mulheres devem ser posicionadas em decúbito lateral ou lateral com inclinação (Abitbol, 1978; Probst e Webb, 1983).

Abitbol (1978) concluiu que a síndrome supina em cães não é causada somente pela compressão da veia cava caudal, ou seja, decorrente unicamente do decúbito adotado. Probst e Webb (1983) também não observaram a ocorrência da síndrome supina em cadelas de 9 a $16 \mathrm{~kg}$, com $60 \pm 2$ dias de gestação, posicionadas em decúbito dorsal e induzidas com tiamilal sódico, e cuja manutenção foi realizada com halotano.

A hipotensão observada deve-se aos efeitos dos fármacos empregados e não ao posicionamento em decúbito dorsal, pois tanto a acepromazina como o propofol e o sevofluorano, promovem diminuição da pressão arterial (Farver et al., 1986; Brock, 1994; Thurmon et al., 1996).

A FCf média elevou-se gradativamente ao longo do procedimento anestésico, sendo o valor 
encontrado em M3 superior ao obtido em M0 (Tab.1). Porém, biologicamente essa elevação não possui significado clínico pois representa um aumento de $4,9 \%$.

A homeostase fetal e a sobrevivência neonatal dependem da manutenção da circulação úteroplacentária. $\mathrm{O}$ feto é vulnerável às alterações no sistema cardiovascular da mãe porque o fluxo sangüíneo fetal não é auto-regulável e a perfusão uterina é diretamente proporcional à pressão arterial e inversamente proporcional à resistência vascular uterina (Robertson e Moon, 2003).

A anestesia obstétrica pode diminuir o fluxo sangüíneo uterino e contribuir para a redução da viabilidade fetal devido à hipotensão materna provocada pelos anestésicos (Thurmon et al., 1996), portanto, deve-se evitar a ocorrência de hipotensão, que pode causar efeitos adversos ao feto (Robertson e Moon, 2003). Contudo, mesmo com a diminuição da PA materna, não se observaram alterações na freqüência cardíaca fetal que pudessem reduzir a viabilidade dos fetos. Nestes, o sistema nervoso simpático é imaturo e desenvolve-se somente após o nascimento. Como conseqüência, o coração é incapaz de aumentar a força de contração, sendo o débito cardíaco estritamente dependente da freqüência cardíaca (Pascoe e Moon, 2001).

Segundo Pascoe e Moon (2001), a FCf acima de $200 \mathrm{bpm}$ nos fetos é indicativo de débito cardíaco adequado, sugerindo, ainda, que valores inferiores necessitam intervenção médica. Porém, esses valores são válidos apenas para filhotes de mães não-anestesiadas. Portanto, os valores de FCf entre 182 e $191 \mathrm{bpm}$, em média, observados neste experimento podem ser usados como referência no caso de utilização do protocolo anestésico com o sevofluorano em cadelas no terço final de gestação.

Concluiu-se que o protocolo anestésico, em cadelas hígidas com 45 dias de gestação, provocou diminuição da pressão sangüínea arterial materna, porém não causou depressão fetal, podendo ser utilizado com segurança no caso de intervenção cirúrgica e/ou anestésica durante esse período gestacional.

\section{REFERÊNCIAS BIBLIOGRÁFICAS}

ABITBOL, M.M. Inferior vena cava compression in the pregnant dog. Am. J. Obstet. Gynecol., v.130, p.194-198, 1978.

BENSON, G.J.; THURMON, J.C. Anesthesia for cesarean section in the dog and cat. Mod. Vet. Pract., v.65, p.29-32, 1984.

BENSON, G.J.; THURMON, J.C. Special anesthetic considerations for caesarean section. In: SHORT C.E. Principles and practice of veterinary anesthesia, Baltimore: Williams \& Wilkins, 1987. p.337-348.

BERNARD, J.M.; WOUTERS, P.F.; DOURSOUT, M.F. et al. Effects of sevoflurane and isoflurane on cardiac and coronary dynamics in chronically instrumented dogs. Anesthesiology, v.72, p.659-662, 1990.

BROCK, N. Acepromazine revised. Can. Vet. J., v.35, p.458-459, 1994.

BROOKS, V.L.; KEIL, L.C. Hemorrhage decreases arterial pressure sooner in pregnant compared with nonpregnant dogs: Role of barorreflex. Am. J. Physiol. 266: H 1610- 1619, 1994.

FARVER, T.B.; HASKINS, S.C.; PATZ, J.D. Cardiopulmonary effects of acepromazine and of the subsequent administration of ketamine in the dog. Am. J. Vet. Res., v.47, p.631-635, 1986.

FELDMAN, E.C.; NELSON, R.W. Breeding, pregnancy and parturition. In: . Canine and feline endocrinology and reproduction. 2.ed. Philadelphia: W.B Saunders, 1996. p.559.

FUNKQUIST, P.E.; NYMAN, G.C.; LOFGREN, A.M.J.et al. Use of propofolisoflurane as an anesthetic regimen for cesarean section in dogs. J. Am. Vet. Med. Assoc., v.211, p.313-317, 1997.

GONÇALVES, R.C. Estudo comparativo entre a acepromazina, clorpromazina e levomepromazina em diferentes doses, através do exame bispectral, termo e presso-algimetria, em cães. 2003. 108f. Dissertação (Mestrado em Anestesiologia Experimental) - Faculdade de Medicina de Botucatu, Universidade Estadual Paulista, Botucatu. 
GUEDEL, A.E. Inhalation anesthesia. 2.ed. New York: MacMillan. 1951.

HALL, L.W.; CLARKE, K.W.; TRIM, C.M. Anaesthesia for obstetrics In: Veterinary anaesthesia. 10.ed. London: W.B. Saunders, 2001. p.481-491.

KAZAMA, T.; IKEDA, K. The comparative cardiovascular effects and induction time of sevoflurane with isoflurane and halothane in dog. Anesthesiology, v.63, p.A17, 1985.

MUTOH, T.; NISHIMURA, R.; KIM, H. et al. Cardiopulmonary effects of sevoflurane, compared with halothane, enflurane and isoflurane in dogs. Am. J. Vet. Res., v.58, p.885890, 1997.

NUNES, N. Monitoração da anestesia. In: FANTONI, D.T.; CORTOPASSI, S.R.G. Anestesia em cães e gatos. São Paulo: Roca, 2002. p.64-81.
PASCOE, P.J.; MOON, P.F. Periparturient and neonatal anesthesia. Vet. Clin. N. Am.: Small Anim. Pract., v.31, p .315-341, 2001.

PROBST, C.W.; WEBB, A.I. Postural influence on systemic blood pressure, gas exchange, and acid/base status in the term-pregnant bitch during general anesthesia. Am. J. Vet. Res., v.44, p.1963-1965, 1983.

ROBERTSON, S.A.; MOON, P.F. Anesthetic management for cesarean section in bitches. Vet. Med., v.98, p.675-696, 2003.

THURMON, J.C.; TRANQUILLI W.J.; BENSON, G.J. Anesthesia for special patients: cesarean section patients. In: Lumb and Jones' veterinary anesthesia, 3.ed. Baltimore: Williams \& Wilkins, 1996. p.818-828.

ZAR, J.H. Biostatistical analysis. 4.ed. New Jersey: Prentice Hall, 1999. 875p. 